Preprint typeset in JHEP style - HYPER VERSION

\title{
A light CP-odd Higgs boson and the muon anomalous magnetic moment
}

\author{
John F. Gunion \\ Department of Physics, University of California, Davis, CA 95616
}

\begin{abstract}
We amalgamate the many experimental limits on the $a b \bar{b}$ coupling of a light CP-odd Higgs boson, $a$, including model-dependence coming from the ratio of the at $\bar{t}$ to the $a b \bar{b}$ coupling. We then employ these limits to analyze the extent to which a light $a$ can make a significant contribution to the discrepancy, $\Delta a_{\mu}$, between the experimentally observed $a_{\mu}$ and that predicted by the standard model. In a "model-independent" framework and in the context of a general two-Higgs-doublet model this is a significant possibility. In contrast, the minimal supersymmetric model is too strongly constrained (after combining experimental and theoretical input) to allow a CP-odd- $a$ explanation of $\Delta a_{\mu}$. The nextto-minimal supersymmetric model allows more freedom and the light $a$ of the model could explain the full $\Delta a_{\mu}$ if $9.2 \mathrm{GeV}<m_{a}<12 \mathrm{GeV}$, or contribute substantially for larger $m_{a}$, if $\tan \beta$ is large.
\end{abstract}


There have been numerous studies [1, 2, 3, 4, 5, 6, 7, 8, 9, 10, 11, 12] of the extent to which the Higgs sector could contribute to the anomalous magnetic moment of the muon, $a_{\mu}$, with current focus on whether it could be used to explain some portion of the now $\sim 3 \sigma$ positive deviation of $a_{\mu}$ with respect to the Standard Model (SM) prediction. The numerical deviation is variously quoted as $\Delta a_{\mu} \sim(27.5 \pm 8.4) \times 10^{-10}$ [13] or $(27.7 \pm 9.3) \times 10^{-10}$ [12]. It is becoming increasingly likely that this deviation can only be explained by new physics of some kind and a beyond-the-standard-model Higgs sector has always been a prime candidate.

Precision electroweak data and direct LEP limits on a light CP-even scalar suggest that it should have SM-like couplings and substantial mass, in which case its contribution to $a_{\mu}$ will only be of order $f e w \times 10^{-11}$. Thus, we will focus on the possible contribution, $\delta a_{\mu}$, of a light CP-odd Higgs boson, $a$, of a CP-conserving Higgs sector, for which it is critical [1] to include the two-loop Barr-Zee type diagrams [14] since the one-loop $a_{\mu}$ contribution is negative whereas the two-loop contribution is positive in popular models.

Of particular interest is the $m_{a}<2 m_{b}$ region, for which a light Higgs, $h$, with SM-like $W W, Z Z$ and fermionic couplings can have mass $m_{h} \sim 100 \mathrm{GeV}$ while still being consistent with LEP data by virtue of $h \rightarrow a a \rightarrow 4 \tau$ decays being dominant 15, 16, 17, 18] (see also [19, 20]). Such a Higgs provides perfect agreement with the rather compelling precision electroweak constraints, and for $B R(h \rightarrow a a) \gtrsim 0.75$ also provides an explanation for the $\sim 2.3 \sigma$ excess observed at LEP in $e^{+} e^{-} \rightarrow Z b \bar{b}$ in the region $M_{b \bar{b}} \sim 100 \mathrm{GeV}$. We term this the "ideal" Higgs scenario. More generally, we will only consider models for which the $Z Z h$ coupling is SM-like (implying zero $Z h a$ coupling and therefore no lower limits on $m_{a}$ coming from $e^{+} e^{-} \rightarrow h a$ at LEP) and $m_{h}$ is such as to give good agreement with precision electroweak data.

Possible contributions to $a_{\mu}$ by the $a$ depend crucially on the $a \mu^{-} \mu^{+}, a b \bar{b}$ and $a t \bar{t}$ couplings defined via

$$
\mathcal{L}_{a f \bar{f}} \equiv i C_{a f \bar{f}} \frac{i g_{2} m_{f}}{2 m_{W}} \bar{f} \gamma_{5} f a .
$$

We assume a Higgs model in which $C_{a \mu^{-} \mu^{+}}=C_{a \tau^{-} \tau^{+}}=C_{a b \bar{b}}$, as typified by a twoHiggs-doublet model (2HDM) of either type-I or type-II (a 2HDM contains Higgs bosons $h, H$ with $m_{H}>m_{h}, a$ and $h^{+}$), or more generally if the lepton and quark masses are generated by the same combination of Higgs fields. (Much larger values of $a_{\mu}$ relative to those we find below are possible in models in which $r=\left(C_{a \mu^{-} \mu^{+}}=C_{a \tau^{-} \tau^{+}}\right) / C_{a b \bar{b}} \gg 1-$ such models include those in which the muon and tau masses are generated by different Higgs fields than the $b$ mass. For $r \neq 1$, our results for $\delta a_{\mu}$ should be rescaled by $r$.) In a $2 \mathrm{HDM}$ of type-II and in the MSSM, $C_{a \mu^{-} \mu^{+}}=C_{a b \bar{b}}=\tan \beta$ (where $\tan \beta=h_{u} / h_{d}$ is the ratio of the vacuum expectation values for the doublets giving mass to up-type quarks vs. down-type quarks) and $C_{a t \bar{t}}=\cot \beta$. In the NMSSM the expressions for $C_{a \mu^{-} \mu^{+}}=C_{a b \bar{b}}$ and $C_{a t \bar{t}}$ include an additional factor discussed later. In a type-I 2HDM, $C_{a \mu^{-} \mu^{+}}=C_{a \tau^{-} \tau^{+}}=C_{a b \bar{b}}=-C_{a t \bar{t}}=-\cot \beta$. In the most general Higgs model, $C_{a \mu^{-} \mu^{+}}$, $C_{a \tau^{-} \tau^{+}}, C_{a b \bar{b}}$ and $C_{a t \bar{t}}$ will be more complicated functions of the vevs of the Higgs fields and the structure of the Yukawa couplings. In this paper, we assume $C_{a \mu^{-} \mu^{+}}=C_{a \tau^{-} \tau^{+}}=C_{a b \bar{b}}$ but allow for general values of $R_{b / t}^{2} \equiv C_{a b \bar{b}} / C_{a t \bar{t}}$. We consider only positive values of $R_{b / t}^{2}$ 
since only these are of relevance for explaining the observed positive $\Delta a_{\mu}$ and positive values are typical of most models.

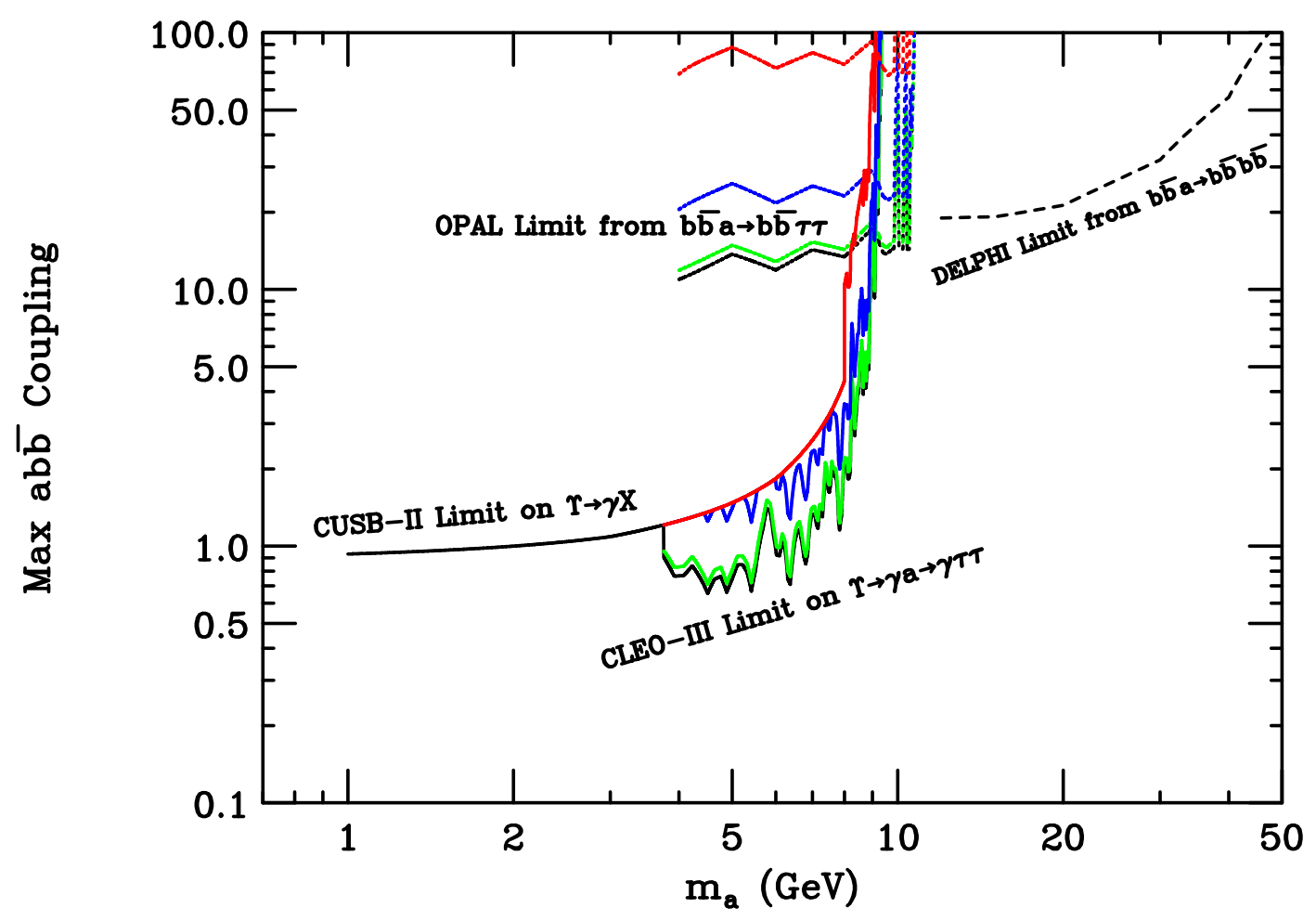

Figure 1: Upper limit, $C_{a b \bar{b}}^{\max }$, on $\left|C_{a b \bar{b}}\right|$ as a function of $m_{a}$ coming directly from experimental data. In the case of limits based on $a \rightarrow \tau^{+} \tau^{-}$, curves for $R_{b / t}=0.5$ (red), 1 (blue), 2 (green), 10 (black) are shown with $R_{b / t}=10$ giving the lowest curves and $R_{b / t}=0.5$ giving the highest curves.

Limits on $\left|C_{a b \bar{b}}\right|$ come from $\Upsilon \rightarrow \gamma a$ decays at $B$ factories and $e^{+} e^{-} \rightarrow b \bar{b} a$ production at LEP. For $m_{a}<2 m_{\tau}$ the strongest limits come from the old (90\% CL - all other limits employed here are 95\% CL) CUSB-II limits [21] on $B R(\Upsilon \rightarrow \gamma X)$, where $X$ is assumed to be visible. For $2 m_{\tau}<m_{a}<9.2 \mathrm{GeV}$, the recent CLEO-III [22] limits on $\Upsilon \rightarrow \gamma a \rightarrow \gamma \tau \tau$ are the strongest (in interpreting these limits one must account for the value of $B R\left(a \rightarrow \tau^{+} \tau^{-}\right.$) - this in turn depends on $R_{b / t}$, but very weakly for $R_{b / t} \geq 2$, see below). For $9.2 \mathrm{GeV}<$ $m_{a}<m_{\Upsilon}$, mixing of the $a$ with various $\eta_{b}$ and $\chi_{0}$ bound states becomes crucial [23]. Ref. [22] gives results for $C_{a b \bar{b}}^{\max }$ in this $m_{a}$ range without taking this mixing into account but notes that their limits cannot be relied upon for $m_{a}>9.2 \mathrm{GeV}$. Whether additional limits can be extracted from lepton non-universality studies in the $9.2<m_{a}<m_{\Upsilon}$ region is being studied [24]. OPAL limits [25] (which assume $B R\left(a \rightarrow \tau^{+} \tau^{-}\right)=1$ ) on $e^{+} e^{-} \rightarrow b \bar{b} \tau \tau$ become numerically relevant for roughly $9 \mathrm{GeV}<m_{a}<2 m_{b}$. Ref. [25] converts these limits to limits on the $a b \bar{b}$ coupling using the modeling of [23]. These are the only limits in the $m_{\Upsilon}<m_{a}<2 m_{b}$ range and continue to be relevant up to $12 \mathrm{GeV}$. Above $m_{a}=2 m_{b}$ these $a b \bar{b}$ coupling limits become quite weak due to the $\eta_{b}-a$ mixing and the decrease of $B R(a \rightarrow \tau \tau)$. For $m_{a} \geq 12 \mathrm{GeV}$, limits on the $a b \bar{b}$ coupling can be extracted from 
$e^{+} e^{-} \rightarrow b \bar{b} a \rightarrow b \bar{b} b \bar{b}$ [26]. The maximum value of $\left|C_{a b \bar{b}}\right|$ allowed by all these various limits, $C_{a b \bar{b}}^{\max }$, is shown in Fig. 1 as a function of $m_{a}$ for several values of $R_{b / t}\left(R_{b / t}=0.5,1,2,10\right)$. Note that there is almost no dependence of $C_{a b \bar{b}}^{\max }$ on $R_{b / t}$ for $R_{b / t} \geq 2$. Values of $\left|C_{a b \bar{b}}\right|$ above 50 raise issues of non-perturbativity of the $a b \bar{b}$ coupling and are likely to be in conflict with Tevatron limits on $b \bar{b} a$ production [27]. $C_{a b \bar{b}}^{\max }$ depends on $R_{b / t}$ when the CLEO-III $\Upsilon \rightarrow \gamma a \rightarrow \gamma \tau^{+} \tau^{-}$or OPAL $b \bar{b} a \rightarrow b \bar{b} \tau^{+} \tau^{-}$limits are the most relevant. What is new in this paper is the systematic incorporation of the $R_{b / t}$ dependence of $C_{a b \bar{b}}^{\max }$ and the systematic incorporation of the $C_{a b \bar{b}}^{\max }$ limits in the context of predictions for $\delta a_{\mu}$ in a wide class of models.

In the case of the simple $2 \mathrm{HDM}(\mathrm{II})$, where $C_{a b \bar{b}}=R_{b / t}=\tan \beta$, values of $m_{a}$ for which $\tan \beta>C_{a b \bar{b}}^{\max }(\tan \beta)$ are not allowed in the model context. These disallowed regions typically emerge in the range $m_{a}<8 \mathrm{GeV}$ for $\tan \beta=1$ rising to $m_{a} \lesssim 10 \mathrm{GeV}$ for higher $\tan \beta$; at higher $\tan \beta$ values they have a complicated structure that we will discuss later. In addition, a disallowed region also arises over a limited $m_{a}$ range starting from $m_{a}>12 \mathrm{GeV}$ when $\tan \beta \gtrsim 18$, the larger the value of $\tan \beta$ the larger the interval. For example, for $\tan \beta=50$ the DELPHI limits imply that the $2 \mathrm{HDM}(\mathrm{II})$ is not consistent for $12 \lesssim m_{a} \lesssim 37 \mathrm{GeV}$ and the OPAL and Upsilon limits imply that the $2 \mathrm{HDM}(\mathrm{II})$ is not consistent for $m_{a}<10 \mathrm{GeV}$. In contrast, for $\tan \beta=10$ the $2 \mathrm{HDM}(\mathrm{II})$ model is always consistent with the DELPHI limits and is only inconsistent (with CLEO-III and CUSB limits) for $m_{a} \lesssim 9 \mathrm{GeV}$. These $2 \mathrm{HDM}$ (II) results are an update of the results obtained in [8]. The results in all other models, in particular in the NMSSM context are new. ${ }^{1}$

We will now explore the implications for $a_{\mu}$. Since the two-loop contributions include that with a $t$-loop as well as those with $b$ and $\tau$ loops, we must specify the value of $C_{a t \bar{t}}$ relative to $C_{a b \bar{b}}$ in order to compute the contribution of $a$ to $a_{\mu}$ for a given $C_{a b \bar{b}}$ value. In a $2 \mathrm{HDM}$ of type-II, including the MSSM and NMSSM, $C_{a t \bar{t}}=\cot \beta$ and after including the two-loop diagrams $\delta a_{\mu}>0$ for $m_{a}>2.6,2,0 \mathrm{GeV}$ if $\tan \beta>5,3,1$. In a type-I 2HDM, $C_{a t \bar{t}}=-C_{a b \bar{b}}=\cot \beta$. Then, the (dominant) top-loop Barr-Zee type diagram gives a negative contribution to $a_{\mu}$ and $\delta a_{\mu}$ is negative for all $m_{a}$. Only models with positive $R_{b / t}^{2}$ are of relevance for explaining the observed positive $\Delta a_{\mu}$. Results for $\delta a_{\mu}$ employing the $C_{a b \bar{b}}=C_{a b \bar{b}}^{\max }$ limits as a function of $m_{a}$ and $R_{b / t}$ and taking $R_{b / t}=1,3,10$ and 50 are plotted in Fig. 2. (For $R_{b / t}<1$, simply multiply the $R_{b / t}=1$ curve by $1 / R_{b / t}^{2}$.)

To a good approximation, $R_{b / t} \geq 50$ is equivalent to dropping the two-loop diagram containing the top quark and gives the smallest result. Since (for positive $C_{a b \bar{b}} / C_{a t \bar{t}}$ ) the two-loop top diagram enters with the same (positive) sign as the $b$ and $\tau$ two-loop diagrams, the largest $\delta a_{\mu}$ values are obtained for the smallest $R_{b / t}$ when using upper limits on the $a b \bar{b}$ coupling as input. As a result, we see in Fig. 2 that for lower $R_{b / t}$ values $\left(1<R_{b / t} \lesssim 3\right)$ any value of $m_{a} \gtrsim 9 \mathrm{GeV}$ would make it possible to obtain $\delta a_{\mu}=\Delta a_{\mu} \sim 27.5 \times 10^{-10}$ for some choice of $C_{a b \bar{b}} \leq C_{a b \bar{b}}^{\max }$. For $R_{b / t}<0.2$, for which $C_{a t \bar{t}}$ enters non-perturbative territory, $\delta a_{\mu}>\Delta a_{\mu}$ if $C_{a b \bar{b}}=C_{a b \bar{b}}^{\max }$ for all $m_{a}$ so that agreement could always be obtained for some $C_{a b \bar{b}}<C_{a b \bar{b}}^{\max }$. However, for $R_{b / t} \gtrsim 10$ the full discrepancy can only be explained if $10 \mathrm{GeV}<m_{a}<12 \mathrm{GeV}$ or $m_{a} \gtrsim 36 \mathrm{GeV}$. Recall, however, that the value of $C_{a b \bar{b}}^{\max }$

\footnotetext{
${ }^{1}$ Several months after arXiv submission of this paper, similar results for the NMSSM were obtained in 28.
} 


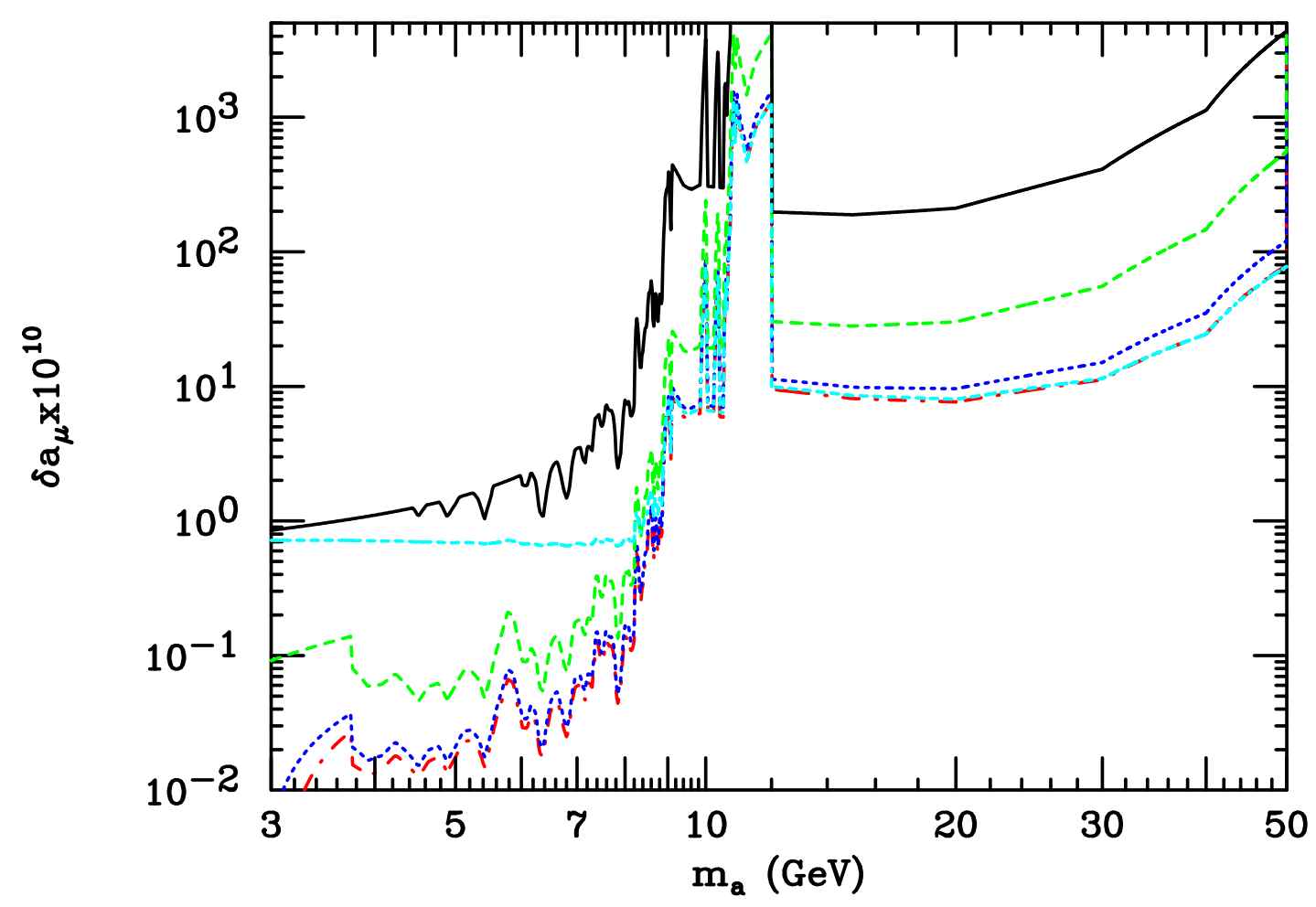

Figure 2: The value of $\delta a_{\mu}$ from CP-odd $a$ loops is plotted as a function of $m_{a}$ for $R_{b / t}=1$ (black, solid), $R_{b / t}=3$ (green, dashes), $R_{b / t}=10$ (blue, dots) and $R_{b / t}=50$ (red, long dash, short dot, lowest curve), assuming maximal $a b \bar{b}$ coupling, $C_{a b \bar{b}}^{\max }$, from Fig. 1. Also shown as the dotdash cyan curve is the $2 \mathrm{HDM}(\mathrm{II})$ prediction for $\tan \beta=C_{a b \bar{b}}^{\max }(\tan \beta)$, i.e. the largest possible (self-consistent) choice of $\tan \beta$ within the $2 \mathrm{HDM}(\mathrm{II})$ context. For $m_{a} \gtrsim 9 \mathrm{GeV}$ this latter curve coincides with the $R_{b / t}=50$ curve.

extracted from the data in the former region relies on the modeling for the $a-\eta_{b}$ mixing employed in the experimental analysis. Also, for $m_{a}>36 \mathrm{GeV}$ and $R_{b / t} \geq 10, \delta a_{\mu}=\Delta a_{\mu}$ requires non-perturbative $C_{a b \bar{b}}>50$ values.

Of course, it is interesting to know what value of $C_{a b \bar{b}}<C_{a b \bar{b}}^{\max }$ is needed in order to match the observed $\Delta a_{\mu}=27.5 \times 10^{-10}$ for those $m_{a}$ and $R_{b / t}$ values for which this is possible. The results for the general case in which $C_{a b b}$ is not correlated with $R_{b / t}$ are plotted in Fig. 3. In general, for low values of $R_{b / t}$ (for which the top loop is a major contributor to $\left.\delta a_{\mu}\right)$ rather modest values of $C_{a b \bar{b}}$ will reproduce the observed $\Delta a_{\mu}$. As $R_{b / t}$ increases, the bottom loop diagrams must reproduce $\Delta a_{\mu}$ on their own and increasingly large values of $C_{a b \bar{b}}$ are required. As we shall see, one particularly interesting range of $m_{a}$ for $R_{b / t} \geq 10$ is $9.9 \mathrm{GeV} \lesssim m_{a} \lesssim 12 \mathrm{GeV}$. In Fig. 3, we see that in this $m_{a}$ range the observed $\Delta a_{\mu}=27.5 \times 10^{-10}$ is matched for $C_{a b \bar{b}}$ in the range $28 \leq C_{a b \bar{b}} \leq 32$ for $R_{b / t} \geq 10$ when $9.9 \mathrm{GeV} \lesssim m_{a} \lesssim 12 \mathrm{GeV}$.

The above results are modified in the context of more restrictive models. Fig. 4 shows the results for $\delta a_{\mu}$ in the type-II $2 \mathrm{HDM}$, in which $C_{a b \bar{b}}=R_{b / t}=\tan \beta$, obtained for various $\tan \beta$ values. In the type-II $2 \mathrm{HDM}$, the value of $\delta a_{\mu}$ is determined once $\tan \beta$ and $m_{a}$ 


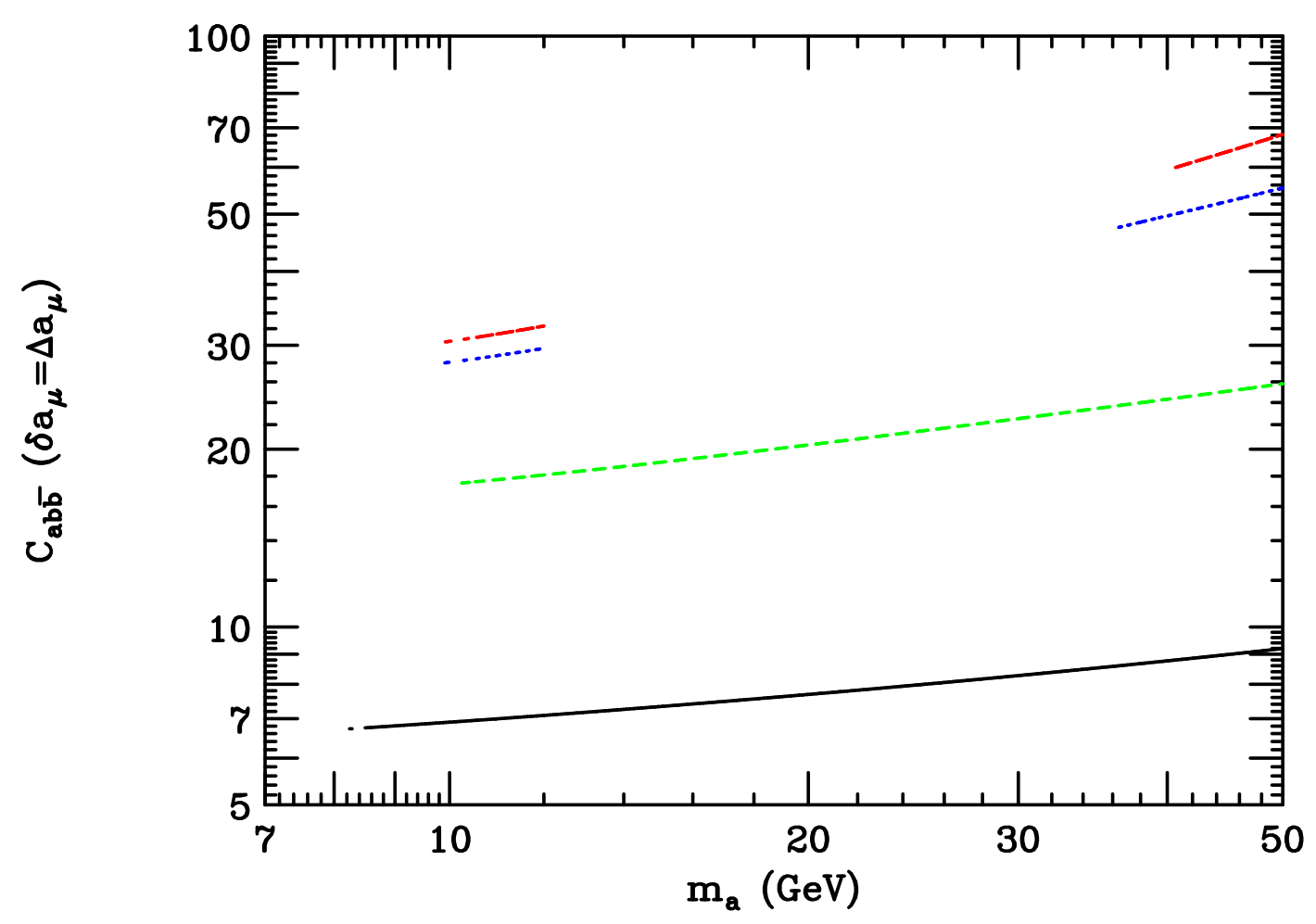

Figure 3: The value of of $C_{a b \bar{b}}$ required in order that $\delta a_{\mu}=27.5 \times 10^{-10}$ is plotted as a function of $m_{a}$ for $R_{b / t}=1$ (black, solid), $R_{b / t}=3$ (green, dashes), $R_{b / t}=10$ (blue, dots) and $R_{b / t}=50$ (red, long dash, short dot, highest curve), for those choices of $m_{a}$ such that the required $C_{a b \bar{b}}$ is less than $C_{a b \bar{b}}^{\max }$ as plotted in Fig. 1. Gaps for any given $R_{b / t}$ curve correspond to $m_{a}$ values for which $C_{a b \bar{b}}>C_{a b \bar{b}}^{\max }$ would be required.

are specified. Unlike the very general case just considered, for which $R_{b / t}$ is not related to $C_{a b \bar{b}}$, in the $2 \mathrm{HDM}(\mathrm{II})$ context one cannot have large $C_{a b \bar{b}}$ without having large $R_{b / t}$, which then minimizes the very important (positive) top loop contribution. Thus, the largest $\delta a_{\mu}$ values are now obtained with large $\tan \beta$ values. The possibilities are also constrained by the requirement that $\tan \beta$ cannot exceed $C_{a b \bar{b}}^{\max }(\tan \beta)$. The gaps in the curves of Fig. 1 are those regions where $\tan \beta>C_{a b \bar{b}}^{\max }(\tan \beta)$. The result is that in order to obtain a value of $\delta a_{\mu}$ of order $27.5 \times 10^{-10}$ that also has $\tan \beta \leq C_{a b \bar{b}}^{\max }(\tan \beta)$ requires a rather precisely fixed value of $\tan \beta \sim 30-32$ and $m_{a} \sim 9.9-12 \mathrm{GeV}$ (see the $\tan \beta=32$ dotdash cyan curve). In the context of the most general CP-conserving type-II $2 \mathrm{HDM}$, any value in the above small range is not excluded using combined $Z h$ and $h a$ LEP data 29] so long as $m_{h} \gtrsim 60 \mathrm{GeV}$; and, there are no limits on $m_{a}$ if $m_{h} \gtrsim 100 \mathrm{GeV}$. Further, contributions to the precision electroweak observables $S$ and $T$ are tiny if $m_{H}=m_{h^{+}}$when $h$ has SM-like $Z Z h$ coupling. As a further remark, we note from trends as $\tan \beta$ increases apparent in Fig. 1 (lower plot) that for $\tan \beta$ values above 50 (i.e. outside the perturbative limit on this coupling) one will not be able to have $\tan \beta<C_{a b \bar{b}}^{\max }(\tan \beta)$ in the $m_{a}<12 \mathrm{GeV}$ zone, but that at some largish value of $m_{a}$ above about $40 \mathrm{GeV}$ one will be able to achieve a 

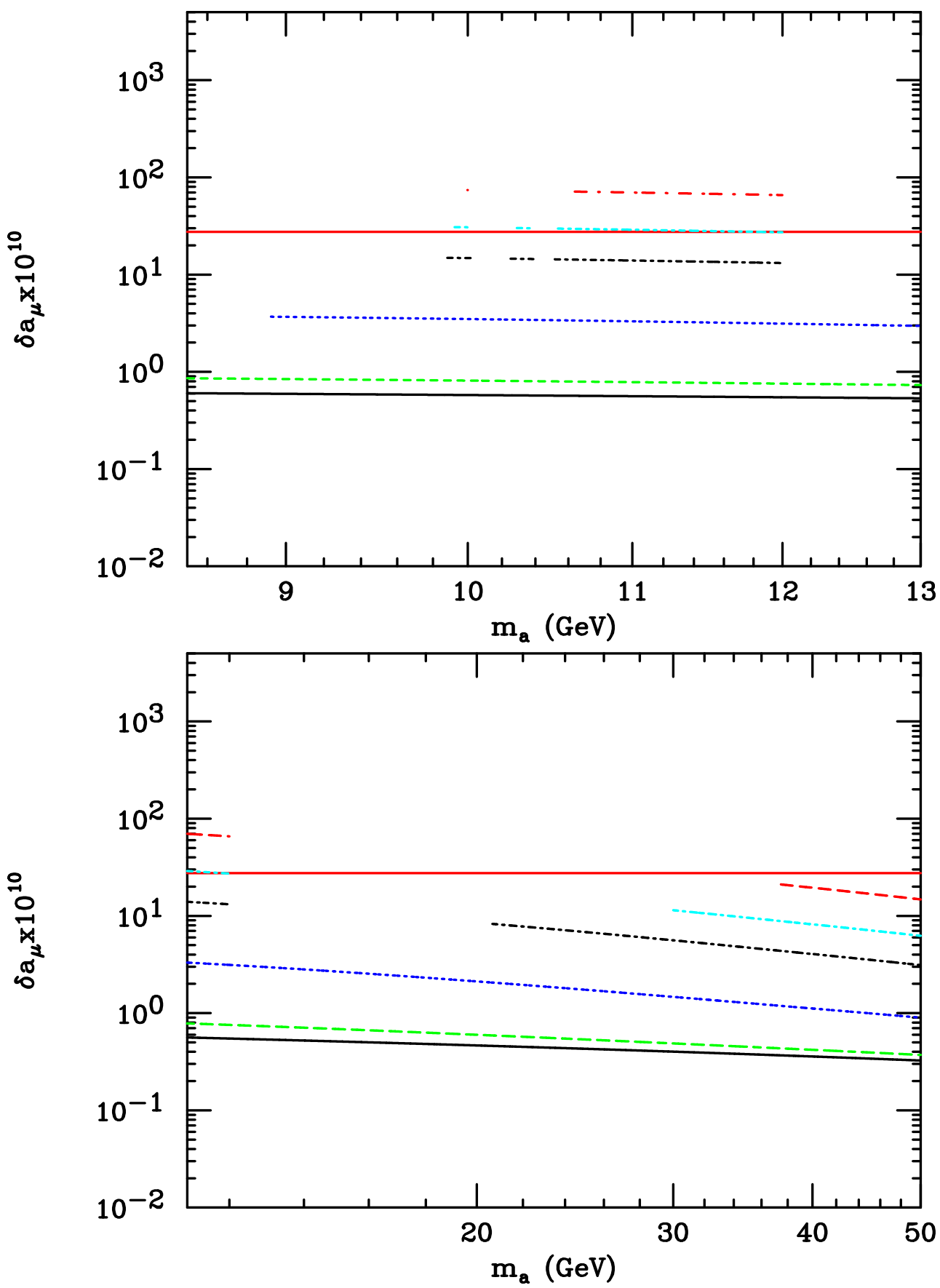

Figure 4: The value of $\delta a_{\mu}$ from CP-odd $a$ loops is plotted as a function of $m_{a}$ for $\tan \beta=1$ (black, solid), $\tan \beta=3$ (green, dashes), $\tan \beta=10$ (blue, dots), $\tan \beta=22$ (black, dotdash), $\tan \beta=32$ (cyan, dotdash) and $\tan \beta=50$ (red, long dash, short dot, highest curve), assuming the $2 \mathrm{HDM}(\mathrm{II})$ model with $R_{b / t}=\tan \beta$ and requiring that $\tan \beta \leq C_{a b \bar{b}}^{\max }(\tan \beta)$. Omitted regions are those for which $\tan \beta>C_{a b \bar{b}}^{\max }(\tan \beta)$ as plotted in Fig. 11. Note the multiple gaps for the $\tan \beta=22,32,50$ cases in the $10 \mathrm{GeV} \leq m_{a} \leq 11 \mathrm{GeV}$ region. An intersection of the solid red line at $\delta a_{\mu}=27.5 \times 10^{-10}$ with a $2 \mathrm{HDM}$ (II) curve essentially only occurs in the $\tan \beta=32$ case.

match to $\Delta a_{\mu}$. This is because the DELPHI limits on $C_{a b \bar{b}}$ deteriorate so rapidly as $m_{a}$ increases above $40 \mathrm{GeV}$. 
As a further perspective on the $2 \mathrm{HDM}$ (II) results, we plot in Fig. 2 the largest possible value of $\delta a_{\mu}$ within the $2 \mathrm{HDM}(\mathrm{II})$ as a function of $m_{a}$ (the dotdash cyan curve). This maximal value is obtained when $\tan \beta=C_{a b \bar{b}}^{\max }(\tan \beta)$ (i.e. for the largest self-consistent choice of $\tan \beta$ such that $C_{a b \bar{b}}=\tan \beta$ ). Again it is apparent that $\delta a_{\mu}$ can match (or exceed) $27.5 \times 10^{-10}$ in the range $9.9 \lesssim m_{a} \lesssim 12 \mathrm{GeV}$. And, to repeat, matching in this range is always achieved for $\tan \beta \sim 30-32$.

The ability to achieve $\delta a_{\mu}=\Delta a_{\mu}$ is much more constrained in the popular Minimal Supersymmetric Model (MSSM). In the MSSM, the LEP lower limit on $m_{a}$ is of order $90-100 \mathrm{GeV}$, depending upon $\tan \beta$ and precise model inputs [30]. For $m_{a}>90 \mathrm{GeV}$, $\delta a_{\mu}=\Delta a_{\mu}$ is only achievable for $C_{a b \bar{b}}=\tan \beta$ well above the upper bound of 50 employed here. (Of course, if the MSSM sparticles are light, their contributions could yield the observed $\Delta a_{\mu}$ [31.)

The Next-to-Minimal Supersymmetric model (NMSSM) provides more fertile ground. The NMSSM is obtained by adding a singlet superfield $\widehat{S}$ to the MSSM Higgs superfields $\widehat{H}_{u}$ and $\widehat{H}_{d}$. Ref. 32] was the first to consider the NMSSM Higgs sector phenomenology in detail. The scalar component of $\widehat{S}$ contains one CP-even and one CP-odd scalar field. The resulting Higgs sector thus contains three CP-even Higgs bosons $\left(h_{1,2,3}\right)$ and two CP-odd Higgs bosons $\left(a_{1,2}\right)$, all of which can have a singlet component. A convenient program for exploring the NMSSM Higgs sector is NMHDECAY [33, 34]. We will not consider contributions to $a_{\mu}$ from sparticles as recently studied in [11, 12].

The NMSSM is especially attractive in that it allows for the "ideal" Higgs sector described earlier with $m_{h_{1}} \sim 100 \mathrm{GeV}$, consistent with LEP data if $m_{a_{1}}<2 m_{b}$ and $B R\left(h_{1} \rightarrow a_{1} a_{1}\right)>0.75$. For $m_{a_{1}}>2 m_{b}$, one must have $m_{h_{1}} \gtrsim 110 \mathrm{GeV}$ to avoid LEP bounds. (But, for $110 \mathrm{GeV} \lesssim m_{h_{1}} \lesssim 163 \mathrm{GeV}$, so long as the $Z Z h_{1}$ coupling is SM-like the agreement with precision electroweak data is still within the $95 \%$ CL limit unless only the "leptonic" determination of $\sin ^{2} \theta_{\ell}^{\text {eff }}$ is employed in the precision electroweak analysis; the latter yields a much higher CL for the overall fit and requires $m_{h_{1}} \lesssim 105 \mathrm{GeV}$ at $95 \%$ CL - see 35] for details).

The most crucial parameter for the NMSSM analysis is $\cos \theta_{A}$ defined by

$$
a_{1}=\cos \theta_{A} a_{M S S M}+\sin \theta_{A} a_{S},
$$

where $a_{M S S M}$ is the CP-odd (doublet) scalar in the MSSM sector of the NMSSM and $a_{S}$ is the additional CP-odd singlet scalar of the NMSSM. In terms of $\cos \theta_{A}, C_{a \mu^{-} \mu^{+}}=C_{a b \bar{b}}=$ $\cos \theta_{A} \tan \beta$ and $C_{a t \bar{t}}=\cos \theta_{A} \cot \beta$.

Before proceeding, we consider possible constraints from precision electroweak data. Since the light SM-like $h_{1}$ already gives good agreement, the rest of the Higgs sector should give a small contribution to $S$ and $T$ (assuming sparticle contributions are not substantial). One finds that if $m_{a_{1}}$ is in the range considered and $h_{1}$ is SM-like, then it is typically the case that either $h_{2}$ or $h_{3}$ is mainly singlet, denoted $h_{S}$, and the other, denoted here as $h_{D}$, is mainly doublet. Further, the $Z h_{S} a_{1}$ coupling is very tiny while the $Z h_{D} a_{1}$ coupling is maximal and $m_{h^{+}} \sim m_{a_{2}} \sim m_{h_{D}}$. With these inputs, one finds that the extra contributions from the Higgs sector to $S$ and $T$ are very small and the excellent agreement with precision electroweak constraints coming from the $h_{1}$ is preserved. 


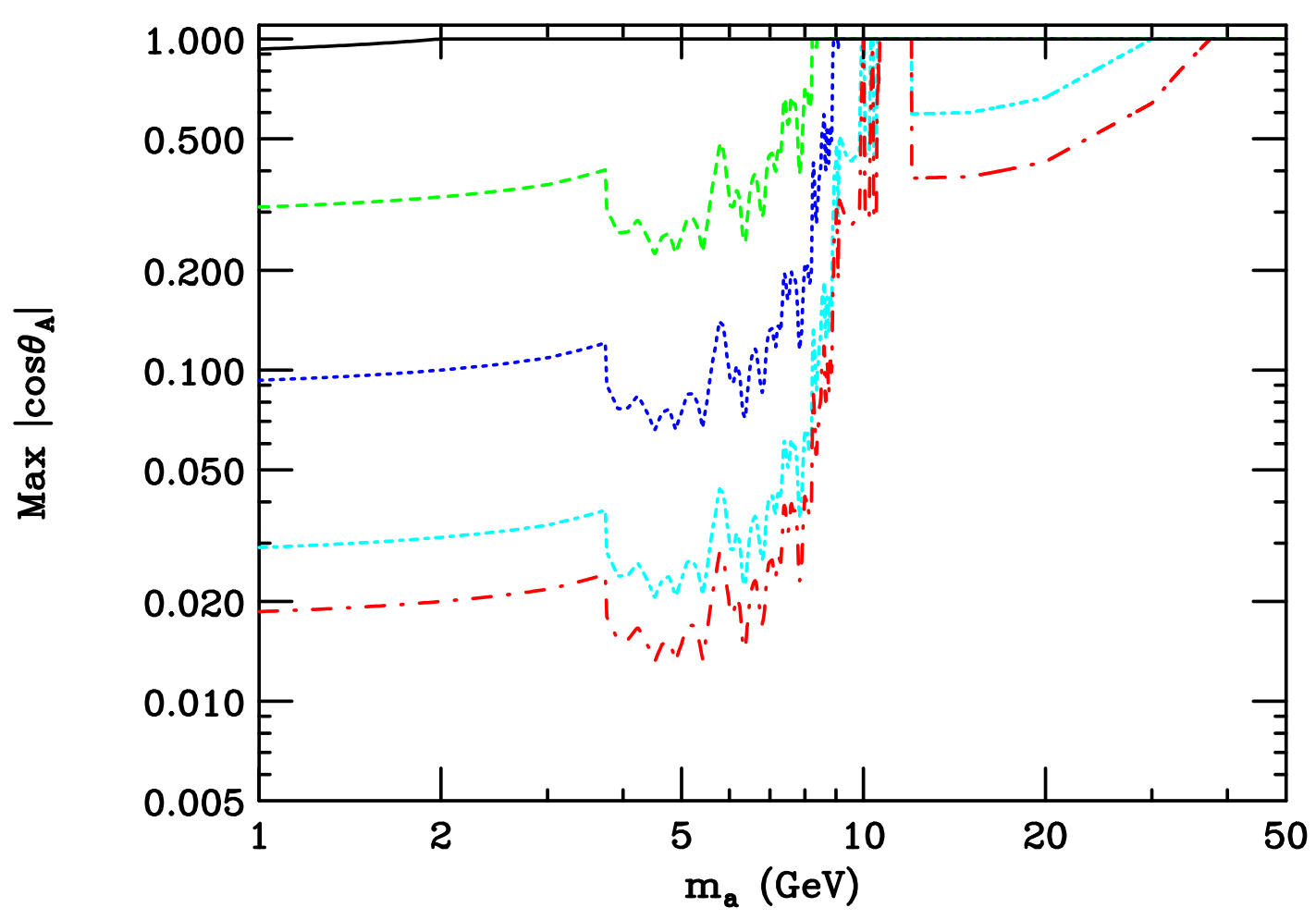

Figure 5: $\cos \theta_{A}^{\max }$ in the NMSSM (where $C_{a b \bar{b}}=\cos \theta_{A} \tan \beta$ ) as a function of $m_{a}$ after requiring $\cos \theta_{A}^{\max } \tan \beta=C_{a b \bar{b}}^{\max }$ using the $C_{a b \bar{b}}^{\max }$ values of Fig. 1. The different curves correspond to $\tan \beta=1$ (black, solid, upper curve), 3 (green, dashes), 10 (blue, dots), 32 (cyan, dotdash) and 50 (red, long dash, short dot, lowest curve).

Let us now consider $a_{1} \equiv a$ contributions to $a_{\mu}$ for various fixed $\tan \beta$ values. Then, $\cos \theta_{A}$ is constrained by the requirement that $C_{a b \bar{b}}=\cos \theta_{A} \tan \beta \leq C_{a b \bar{b}}^{\max }$, which constrains $\cos \theta_{A}$ to very small values for low $m_{a}$ and large $\tan \beta$. However, no matter what the value of $\tan \beta$, the extra freedom of adjusting $\cos \theta_{A}$ does allow us to avoid gaps in $m_{a}$ for which $C_{a b \bar{b}}>C_{a b \bar{b}}^{\max }$. This, in turn, will give us more possibilities for $\delta a_{\mu}$. Inputting the values of $C_{a b \bar{b}}^{\max }$ as a function of $m_{a}$ we obtain the results of Fig. 5 for the maximum allowed value of $\cos \theta_{A}$ as a function of $m_{a}$ for various $\tan \beta$ values.

We now turn to the resulting NMSSM predictions for $a_{\mu}$. The value of $\delta a_{\mu}$ is largest for $\cos \theta_{A}=\cos \theta_{A}^{\max }$. The resulting values of $\delta a_{\mu}$ are plotted as a function of $m_{a}$ in Fig. 6 . As in the generic case, the strong constraints from Upsilon physics imply that significant contributions to $a_{\mu}$ are not possible until $m_{a}$ exceeds roughly $9.2 \mathrm{GeV}$. To understand why $\delta a_{\mu}$ increases with increasing $\tan \beta$ for $m_{a}>12 \mathrm{GeV}$, whereas it decreases with increasing $\tan \beta$ for low $m_{a}$, we first note that the 2-loop, top-loop contribution to $\delta a_{\mu}$ is independent of $\tan \beta$ (because of a $C_{a \mu^{-} \mu^{+}} C_{a t \bar{t}}$ structure that is $\tan \beta$-independent), whereas the 2-loop bottom-loop contribution increases as $\tan ^{2} \beta$ (because of a $C_{a \mu^{-} \mu^{+}} C_{a b \bar{b}} \propto \tan ^{2} \beta$ structure). Numerically, before including the extra $\tan ^{2} \beta$ factor for the latter, the 2-loop, top-loop contribution is much larger than the 2-loop, bottom-loop contribution. Of course, both 


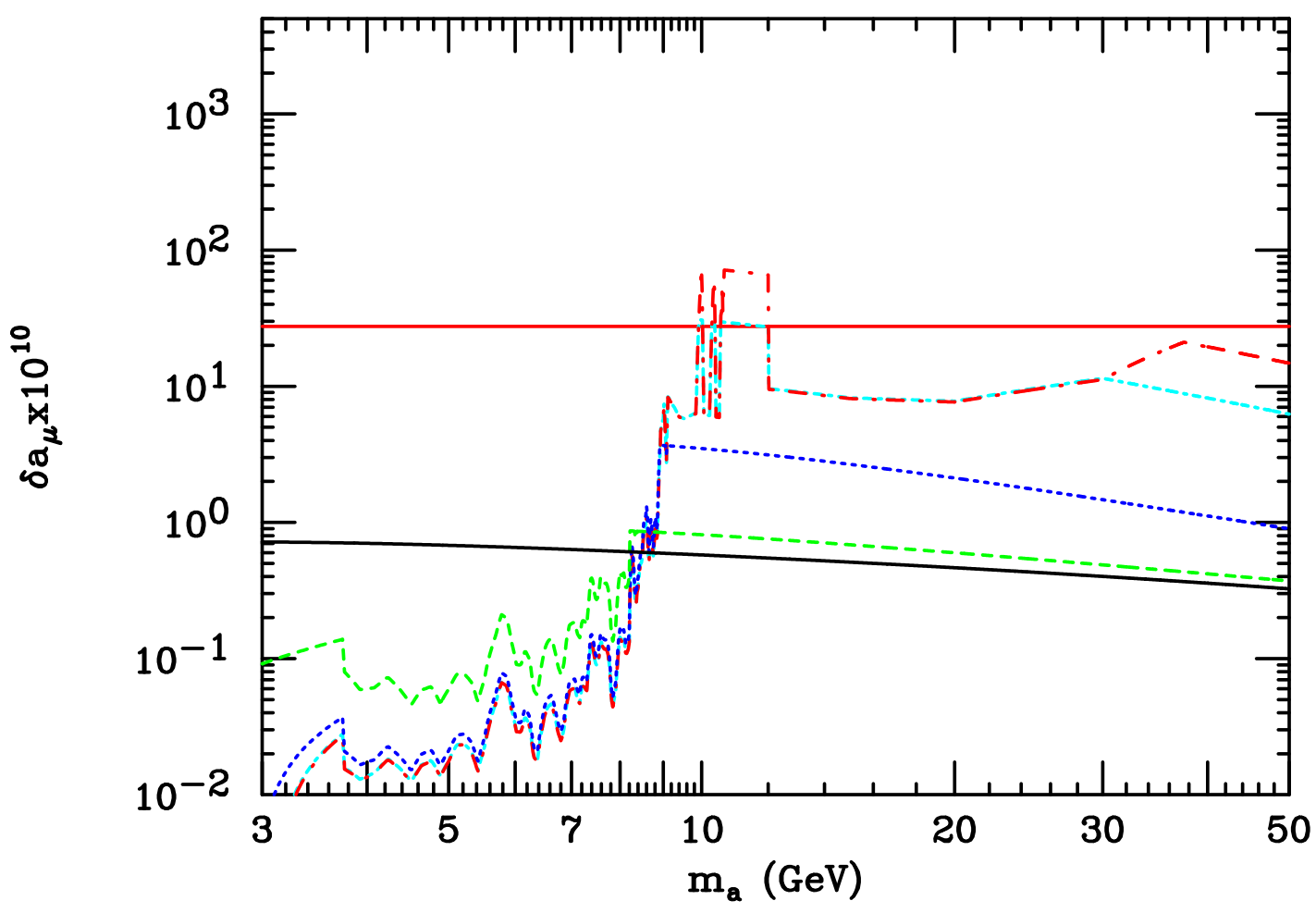

Figure 6: The maximum contribution of the CP-odd $a$ to $a_{\mu}$ as a function of $m_{a}$ in the NMSSM, for which $C_{a b \bar{b}}=\cos \theta_{A} \tan \beta$, after employing $\cos \theta_{A}=\cos \theta_{A}^{\max }$ where $\cos \theta_{A}^{\max }$ is plotted for different $\tan \beta$ values in Fig. 5. Curve notation is as in Fig. 5. The horizontal solid red line is located at $\delta a_{\mu}=27.5 \times 10^{-10}$.

contributions are multiplied by $\left(\cos \theta_{A}\right)^{2}$. Thus, when $C_{a b \bar{b}}^{\max }$ is independent of $\tan \beta$ and $\cos \theta_{A}^{\max }=1$ (as for $m_{a}>12 \mathrm{GeV}$ and $\tan \beta \lesssim 20$ ) the resulting $\delta a_{\mu}$ will always increase with $\tan \beta$. However, at low $m_{a}$, the very strong Upsilon constraints on $C_{a b \bar{b}}$ imply that $\cos \theta_{A}^{\max }$ rapidly decreases with increasing $\tan \beta$ which suppresses the numerically more important 2-loop, top-loop contribution resulting in smaller $\delta a_{\mu}$ as $\tan \beta$ increases.

From Fig. 6, we observe that the maximal $\delta a_{\mu}$ can exceed $\Delta a_{\mu}=27.5 \times 10^{-10}$ for $9.9 \mathrm{GeV} \lesssim m_{a} \lesssim 12 \mathrm{GeV}$ if $\tan \beta \geq 32$, with an almost precise match to this value of $\Delta a_{\mu}$ for $\tan \beta=32$ (or for $\tan \beta$ as low as $\tan \beta=30-$ see the 2 HDM discussion). For $\tan \beta=50$, one can match $\Delta a_{\mu}$ by using a value of $\cos \theta_{A}$ below $\cos \theta_{A}^{\max }$. (As discussed below, the fact that matching is possible for $9.9 \mathrm{GeV} \lesssim m_{a} \lesssim 2 m_{B}$ is particularly interesting in the context of the ideal Higgs scenario.) Further, the maximal $\delta a_{\mu}$ is in the $7-20 \times 10^{-10}$ range for $12 \mathrm{GeV}<m_{a} \lesssim 48 \mathrm{GeV}$ for $\tan \beta=32$ and for $12 \mathrm{GeV}<m_{a} \lesssim 70 \mathrm{GeV}$ for $\tan \beta=50$.

At this point, it is worth stressing the other desirable features of the $m_{h} \sim 100 \mathrm{GeV}$, $m_{a} \lesssim 2 m_{B}, B R(h \rightarrow a a)>0.75$ scenario as discussed in [15, 16, 17, 18]. These references examined the degree to which obtaining the observed value of $m_{Z}$ requires very precisely tuned values of the GUT scale parameters of the MSSM and NMSSM. One finds that in 
any supersymmetric model this finetuning is always minimized for GUT scale parameters that yield a SM-like $h$ with $m_{h} \leq 100 \mathrm{GeV}$, something that is only consistent with LEP data if the $h$ has unexpected decays that reduce the $h \rightarrow b \bar{b}$ branching ratio while not contributing to $h \rightarrow b \bar{b} b \bar{b}$ (also strongly constrained by LEP data). A Higgs sector with a light $a$ for which $B R(h \rightarrow a a)>0.75$ and with $m_{a}$ small enough that $a$ decays to $B \bar{B}$ final states are disallowed (i.e. $m_{a}<10.56 \mathrm{GeV}$ ) provides a very natural possibility for allowing minimal finetuning. The NMSSM provides one possible example.

In conclusion, the combined limits from $\Upsilon$ decays and $b \bar{b} a$ Yukawa production at LEP, along with the perturbativity requirement of $C_{a b \bar{b}}<50$, imply that the entire $a_{\mu}$ discrepancy of $\Delta a_{\mu} \sim 30 \times 10^{-10}$ cannot have a purely Higgs sector explanation without going beyond the MSSM. In the less-constrained NMSSM, achieving $\delta a_{\mu} \sim \Delta a_{\mu}$ requires relatively high $\tan \beta$ and a value of $m_{a}$ between about $10 \mathrm{GeV}$ and $2 m_{B}$. On the one hand, this is a highly motivated $m_{a}$ region in the NMSSM since, as described earlier, it would allow an "ideal" SM-like $h$ with $m_{h} \lesssim 100 \mathrm{GeV}$ decaying mainly via $h \rightarrow a a \rightarrow 4 \tau$. Such an $h$ would escape LEP limits while allowing for low $m_{Z}$-finetuning. However, on the other hand, in the NMSSM $m_{a}<2 m_{B}$ most naturally arises when close to the $U(1)_{R}$ symmetry limit. In this case, the $a$ is mainly singlet, implying that $\cos \theta_{A}$ is small and that $C_{a b \bar{b}}=\cos \theta_{A} \tan \beta$ is typically $\mathcal{O}(1)$ [17], whereas $C_{a b \bar{b}} \sim 30$ is needed to match the observed $\Delta a_{\mu}$.

Nonetheless, the possibility that a CP-odd $a$ with $10 \mathrm{GeV} \lesssim m_{a} \lesssim 12 \mathrm{GeV}$ could explain the $a_{\mu}$ anomaly should be taken seriously, Thus, finding techniques to experimentally probe for an $a$ in the $10 \mathrm{GeV}<m_{a}<12 \mathrm{GeV}$ region should be a high priority. Such new techniques could either end up limiting $C_{a b \bar{b}}$ sufficiently that $\Delta a_{\mu}$ cannot be explained in the $2 \mathrm{HDM}(\mathrm{II})$ or NMSSM frameworks or else actually allow a discovery of a light $a$. Of course, this is a region in which $\eta_{b}-a$ mixing will surely be a complication.

As an aside, one must not forget that in supersymmetric models sparticle loops could have two important roles: (i) they could directly yield large contributions to $a_{\mu}$; and (ii) they could modify the relations between $C_{a \mu^{-} \mu^{+}}, C_{a \tau^{-} \tau^{+}}, C_{a b \bar{b}}$ and $C_{a t \bar{t}}$.

If one goes beyond the MSSM and NMSSM Higgs sectors to the more general type-II 2HDM, then, keeping $C_{a b \bar{b}}<50$, only an $a$ with $10 \mathrm{GeV}<m_{a}<12 \mathrm{GeV}$ with $C_{a b \bar{b}} \sim 30-32$ could give $\delta a_{\mu}=\Delta a_{\mu}$. (A type-I $2 \mathrm{HDM}$ gives negative $\delta a_{\mu}$ that is large for $m_{a}>8 \mathrm{GeV}$ if $C_{a b \bar{b}}=C_{a b \bar{b}}^{\max }$ and is therefore strongly disfavored by the observed positive $\Delta a_{\mu}$.)

Obtaining the observed $\Delta a_{\mu}$ in the most general Higgs model for which the $a b \bar{b}$ coupling magnitude is disconnected from the ratio $R_{b / t}^{2}$ of the $a b \bar{b}$ to $a t \bar{t}$ couplings is generically possible so long as $R_{b / t}^{2}>0$. For $R_{b / t}=1, m_{a}>8 \mathrm{GeV}$ and a relatively modest value of $C_{a b \bar{b}}$ (well below the maximum allowed) will yield $\delta a_{\mu}=\Delta a_{\mu}$. As $R_{b / t}$ increases, the required $C_{a b \bar{b}}$ increases. For larger $R_{b / t}$, there are regions of $m_{a}$ for which the required $C_{a b \bar{b}}$ exceeds the upper experimental bound, $C_{a b \bar{b}}^{\max }$. Further, $\delta a_{\mu}=\Delta a_{\mu}$ cannot be achieved above an $R_{b / t}$-dependent maximum $m_{a}$ if $C_{a b \bar{b}}<50$ is imposed. For $R_{b / t}<0.2$, even very low values of $m_{a}$ will yield the observed $\Delta a_{\mu}$ for an appropriate choice of $C_{a b \bar{b}}<C_{a b \bar{b}}^{\max }$.

\section{Acknowledgments}

This work was supported by U.S. DOE grant No. DE-FG03-91ER40674. This research 
was supported in part by the National Science Foundation under Grant No. PHY05-51164 while at KITP and by the Aspen Center for Physics. JFG thanks R. Dermisek, T. Han, B. McElrath, and P. Osland for their comments on the manuscript.

\section{References}

[1] D. Chang, W. F. Chang, C. H. Chou and W. Y. Keung, Phys. Rev. D 63, 091301 (2001) [arXiv:hep-ph/0009292].

[2] A. Dedes and H. E. Haber, JHEP 0105, 006 (2001) [arXiv:hep-ph/0102297].

[3] K. m. Cheung, C. H. Chou and O. C. W. Kong, Phys. Rev. D 64, 111301 (2001) [arXiv:hep-ph/0103183].

[4] C. H. W. Chen and C. Q. Geng, Phys. Lett. B 511, 77 (2001) [arXiv:hep-ph/0104151].

[5] Y. L. Wu and Y. F. Zhou, Phys. Rev. D 64, 115018 (2001) [arXiv:hep-ph/0104056].

[6] M. Krawczyk, arXiv:hep-ph/0103223.

[7] A. Arhrib and S. Baek, Phys. Rev. D 65, 075002 (2002) [arXiv:hep-ph/0104225].

[8] M. Krawczyk, Acta Phys. Polon. B 33, 2621 (2002) [arXiv:hep-ph/0208076].

[9] K. Cheung and O. C. W. Kong, Phys. Rev. D 68, 053003 (2003) [arXiv:hep-ph/0302111].

[10] O. C. W. Kong, arXiv:hep-ph/0402010.

[11] F. Domingo and U. Ellwanger, JHEP 0712, 090 (2007) [arXiv:0710.3714 [hep-ph]].

[12] F. Domingo and U. Ellwanger, arXiv:0806.0733 [hep-ph].

[13] M. Davier, Nucl. Phys. Proc. Suppl. 169, 288 (2007) [arXiv:hep-ph/0701163].

[14] S. M. Barr and A. Zee, Phys. Rev. Lett. 65, 21 (1990) [Erratum-ibid. 65, 2920 (1990)].

[15] R. Dermisek and J. F. Gunion, Phys. Rev. Lett. 95, 041801 (2005) [arXiv:hep-ph/0502105].

[16] R. Dermisek and J. F. Gunion, Phys. Rev. D 73, 111701 (2006) [arXiv:hep-ph/0510322].

[17] R. Dermisek and J. F. Gunion, Phys. Rev. D 75, 075019 (2007) [arXiv:hep-ph/0611142].

[18] R. Dermisek and J. F. Gunion, Phys. Rev. D 76, 095006 (2007) [arXiv:0705.4387 [hep-ph]].

[19] S. Chang, P. J. Fox and N. Weiner, JHEP 0608, 068 (2006) [arXiv:hep-ph/0511250].

[20] S. Chang, R. Dermisek, J. F. Gunion and N. Weiner, arXiv:0801.4554 [hep-ph].

[21] J. Lee-Franzini, Eloisatron Workshop: Higgs 1989:0269-294 (QCD161:I12:1989).

[22] W. Love, et al.(CLEO), CLNS 08/2033, CLEO 08-16 to be submitted to PRL.

[23] M. Drees and K. i. Hikasa, Phys. Rev. D 41, 1547 (1990).

[24] M. A. Sanchis-Lozano, Mod. Phys. Lett. A 17, 2265 (2002) [arXiv:hep-ph/0206156]. M. A. Sanchis-Lozano, Int. J. Mod. Phys. A 19, 2183 (2004) [arXiv:hep-ph/0307313]. E. Fullana and M. A. Sanchis-Lozano, Phys. Lett. B 653, 67 (2007) [arXiv:hep-ph/0702190]. M. A. Sanchis-Lozano, arXiv:0709.3647 [hep-ph].

[25] G. Abbiendi et al. [OPAL Collaboration], Eur. Phys. J. C 23, 397 (2002) [arXiv:hep-ex/0111010]. 
[26] The Delphi Collaboration, ICHEP 2002, DELPHI 2002-037-CONF-571. I employ Table 20 these are very close to those appearing in the figures of J. Abdallah et al. [DELPHI Collaboration], Eur. Phys. J. C 38, 1 (2004) [arXiv:hep-ex/0410017].

[27] Nils Krumnack (CDF plus D0 results) talk at ICHEP2008.

[28] F. Domingo, U. Ellwanger, E. Fullana, C. Hugonie and M. A. Sanchis-Lozano, arXiv:0810.4736 [hep-ph].

[29] G. Abbiendi et al. [OPAL Collaboration], Eur. Phys. J. C 40, 317 (2005) [arXiv:hep-ex/0408097].

[30] S. Schael et al. [ALEPH Collaboration and DELPHI Collaboration and L3 Collaboration and ], Eur. Phys. J. C 47, 547 (2006) [arXiv:hep-ex/0602042].

[31] J. R. Ellis, S. Heinemeyer, K. A. Olive, A. M. Weber and G. Weiglein, JHEP 0708, 083 (2007) [arXiv:0706.0652 [hep-ph]].

[32] J. R. Ellis, J. F. Gunion, H. E. Haber, L. Roszkowski and F. Zwirner, Phys. Rev. D 39, 844 (1989).

[33] U. Ellwanger, J. F. Gunion and C. Hugonie, JHEP 0502, 066 (2005) [arXiv:hep-ph/0406215].

[34] U. Ellwanger and C. Hugonie, Comput. Phys. Commun. 175, 290 (2006) [arXiv:hep-ph/0508022].

[35] M. S. Chanowitz, arXiv:0806.0890 [hep-ph]. 